\title{
Desarrollo e integración de aplicaciones Excel y software profesional de simulación numérica en una clase al revés en ingeniería hidráulica
}

\author{
Bermúdez Pita, María'; Puertas Agudo, Jerónimo²; Cea Gómez, Luis³; Pena Mosquera, \\ Luís ${ }^{4}$ \\ ${ }^{1}$ Instituto Interuniversitario de Investigación del Sistema Tierra en Andalucía, \\ Universidad de Granada, ORCID 0000-0003-3189-4791 \\ ${ }^{2}$ Escuela Técnica Superior de Ingeniería de Caminos, Canales y Puertos, \\ Universidade da Coruña, ORCID 0000-0001-6502-0799 \\ ${ }^{3}$ Escuela Técnica Superior de Ingeniería de Caminos, Canales y Puertos, \\ Universidade da Coruña, ORCID 0000-0002-3920-0478 \\ ${ }^{4}$ Escuela Técnica Superior de Ingeniería de Caminos, Canales y Puertos, \\ Universidade da Coruña, ORCID 0000-0001-9090-2355
}

\section{RESUMEN}

La clase al revés ('flipped classroom') es una de las metodologías activas que ha adquirido mayor popularidad en el campo de la ingeniería. La implantación de esta metodología requiere diseñar tareas adecuadas, apoyadas en buenos materiales, para que los alumnos trabajen fuera del aula, e integrarlas convenientemente con las actividades desarrolladas en clase. Partiendo de esta premisa, en este trabajo se presenta un conjunto de aplicaciones informáticas para uso docente en asignaturas de grado de ingeniería hidráulica, centradas en el cálculo de flujo en presión y en lámina libre. Se describe cómo se han integrado estas aplicaciones en la programación de aula de la asignatura de Hidráulica e Hidrología I (HH1) del Grado en Tecnología de la Ingeniería Civil de la Universidad de A Coruña, dentro de la estrategia de enseñanza-aprendizaje activa. Entre los resultados positivos obtenidos, cabe destacar la familiarización de los estudiantes con las herramientas que se utilizan en la práctica profesional, lo que repercute además en su motivación. Las aplicaciones desarrolladas están a disposición de la 
comunidad docente y esperamos que puedan ser empleadas en otros centros para la mejora del proceso de enseñanza de la ingeniería hidráulica.

PALABRAS CLAVE: metodologías activas; clase al revés; ingeniería hidráulica; aplicaciones informáticas

\section{CITA RECOMENDADA:}

Bermúdez Pita, María; Puertas Agudo, Jerónimo; Cea Gómez, Luis; Pena Mosquera, Luís (2020): Desarrollo e integración de aplicaciones Excel y software profesional de simulación numérica en una clase al revés en ingeniería hidráulica. En De la Torre Fernández, E. (ed.) (2020). Contextos universitarios transformadores: Boas prácticas no marco dos GID. IV Xornadas de Innovación Docente. Cufie. Universidade da Coruña. A Coruña (págs. 17-32).

DOI capítulo: https://doi.org/10.17979/spudc.9788497497756.017

DOl libro: https://doi.org/10.17979/spudc.9788497497756

\section{ABSTRACT}

The flipped classroom is one of the active teaching-learning methodologies that has become more popular in the field of engineering. The implementation of this methodology requires designing appropriate tasks, supported by good materials, that are to be carried out independently by the students outside the classroom, and that are conveniently integrated with the activities developed in class. Based on this premise, this paper presents a set of computational tools for hydraulic engineering students within an undergraduate curriculum, focused on the calculation pressure flow and free surface flow. It describes how these applications have been introduced into the Hydraulics and Hydrology I course of the Civil Engineering Technology Degree at the University of A Coruña, within an active teaching-learning strategy. The familiarization of students with the tools used in real practice, which also increases their motivation and engagement, is among the first positive results observed. The applications are freely available to the teaching community and we hope that they can be used in other centers to improve the teaching process of hydraulic engineering.

KEY WORDS: active methodologies; flipped classroom; hydraulic engineering; computer applications 


\section{INTRODUCCIÓN}

La incorporación al Espacio Europeo de Educación Superior ha supuesto una transformación profunda en la forma de entender el proceso de docencia-aprendizaje, que ha llevado aparejada una renovación metodológica en las aulas universitarias. La clase al revés ('flipped classroom') es una de las metodologías activas que ha adquirido mayor popularidad en el campo de la ingeniería, ya que crea oportunidades para resolver problemas complejos de forma colaborativa.

En esta metodología pierden protagonismo las clases magistrales, en las que se trabajan los niveles cognitivos más bajos de la taxonomía de Bloom (recordar, comprender). En el tiempo de clase se profundiza en los contenidos con actividades que trabajan los niveles cognitivos de orden superior (aplicar, analizar, evaluar, crear), y que implican por tanto la construcción de nuevo conocimiento (Marqués Andrés 2016). Se busca proporcionar una mejor preparación para la futura actividad profesional, que requiere el desarrollo de habilidades que van más allá de la mera adquisición de conocimiento.

Estos cambios metodológicos han sido aplicados a la asignatura de introducción a la hidráulica en la Escuela de Ingeniería Civil de la Universidade da Coruña (UDC). Se trata de una asignatura de grado de carácter obligatorio, en la que los estudiantes tienen un primer contacto con la ingeniería hidráulica. Cubre el contenido estándar de una introducción a la mecánica de fluidos en ingeniería, y está organizada en los siguientes bloques temáticos:

1. Propiedades de los fluidos e hidrostática

2. Ecuaciones fundamentales de la hidráulica

3. Análisis dimensional y semejanza hidráulica

4. Flujo en presión

5. Flujo en lámina libre

Los dos primeros bloques de la asignatura se cubren de modo adecuado mediante los materiales teóricos y las prácticas de laboratorio habituales (UNESCO 1983), que en este caso se desarrollan en el laboratorio hidráulico docente de la Escuela de Ingeniería Civil. Éstas 
incluyen experiencias sencillas vinculadas con la hidrostática y la flotación, experiencias para visibilizar la ley de conservación de la cantidad de movimiento o la experiencia de Reynolds. En el tercer bloque los alumnos realizan prácticas con modelos a escala de obras hidráulicas en el CITEEC (laboratorio de investigación y desarrollo de la UDC), para comprender cómo se aplica el análisis dimensional y la semejanza al desarrollo de modelos físicos.

Los bloques 4 y 5 , que cubren la parte más aplicada de la asignatura y que en total suponen 2/3 de las horas de clase, se imparten tradicionalmente a través de clases teóricas y la resolución manual de ejercicios prácticos. La práctica profesional vinculada a la ingeniería hidráulica no se puede sin embargo separar del uso de herramientas informáticas. Los métodos manuales de cálculo de redes de tuberías ya no tienen sentido, salvo para apuntalar algunos conceptos básicos. El cálculo del flujo en canales o ríos, hace años circunscrito a geometrías muy sencillas, es ahora asumible para todo tipo de situaciones, y existe una amplia oferta de herramientas profesionales, lo que no obsta para que los conceptos básicos que sirven para analizar de modo crítico los resultados sigan siendo necesarios.

Partiendo de esta premisa, en este artículo se presenta un conjunto de aplicaciones informáticas para uso docente en una asignatura de grado de ingeniería hidráulica, dentro de los bloques temáticos de flujo en presión y flujo en lámina libre. Las aplicaciones permiten implementar una estrategia de enseñanza-aprendizaje activa, que se describe con detalle en la sección 2, y contribuyen asimismo a familiarizar a los estudiantes con las herramientas que se utilizan en la práctica profesional. En la sección 3 se describen las distintas aplicaciones con más detalle, ordenadas de acuerdo con el desarrollo cronológico de la asignatura. Finalmente se analiza la aplicabilidad de la metodología.

\section{METODOLOGÍA DOCENTE}

La estrategia de enseñanza-aprendizaje que ha sido aplicada a la asignatura está basada en el modelo pedagógico de clase al revés. En este modelo la exposición de contenidos 0 instrucción directa se traslada fuera del aula, donde los estudiantes trabajan de manera 
independiente los materiales digitales facilitados por los docentes. Esto permite liberar tiempo de clase, que se invierte en tareas y actividades participativas de carácter práctico, como resolución de problemas o casos prácticos (Berrett 2012). El carácter eminentemente práctico de una asignatura de ingeniería hidráulica incentiva y facilita la implantación de una metodología de este tipo. Además, cabe señalar que existen algunos ejemplos muy recientes de su aplicación exitosa en asignaturas con contenidos similares (Wachs et al. 2018).

La metodología seguida en la asignatura es la siguiente. Los docentes distribuyen el material de cada bloque temático a los estudiantes previamente a la primera clase presencial del bloque, a través del entorno virtual de aprendizaje Moodle. Dicho material incluye una selección de contenidos de otros autores y materiales propios creados en el marco del Grupo de Innovación Educativa en Hidráulica Computacional y Experimental de la Universidade da Coruña (Puertas et al. 2016).

Antes de la clase los estudiantes deben trabajar de forma independientemente los materiales y realizar una serie de tareas que aportarán contenido a la clase. En las primeras sesiones presenciales de cada bloque, las tareas consisten en general en la resolución de pequeñas cuestiones y problemas de forma individual. En las sesiones finales de cada bloque, las tareas son grupales (grupos de 3-4 alumnos) y abarcan tanto los recursos facilitados por los profesores como los aspectos trabajados en las clases presenciales previas. Estas tareas requieren la preparación de materiales propios, que después se pondrán en común con el resto de alumnos, como presentaciones, tutoriales o screencasts.

De esta forma, cada sesión de clase incluye en general las siguientes actividades: (1) Presentación de las tareas previas y ronda de preguntas. Se busca resolver dudas, consolidar el aprendizaje y realizar una evaluación formativa del alumno (Gibbs y Simpson 2005). (2) Resolución de problemas. En la segunda parte de las clases, los estudiantes afianzan los conocimientos teóricos y los aplican a la resolución de problemas, con la ayuda de los compañeros y los profesores. (3) Prácticas de laboratorio. En un gran número de sesiones presenciales se han incluido además prácticas de laboratorio. En cursos anteriores los 
docentes han podido comprobar su utilidad para comprender y reforzar conceptos complejos aprendidos desde un punto de vista teórico. (4) Uso de aplicaciones informáticas, desarrolladas por los docentes 0 de tipo profesional, adaptadas a los conocimientos $y / 0$ las capacidades de los estudiantes. Este último apartado, en ocasiones vinculado con las prácticas de laboratorio, es el objeto fundamental de este artículo.

\section{HERRAMIENTAS INFORMÁTICAS}

Es muy interesante que los alumnos manejen dos tipos de herramientas informáticas: algunas de gran sencillez, cuyas bases de cálculo estén claras y puedan ser perfectamente comprendidas por el alumnado, y que sirvan como refuerzo y aplicación de los conceptos básicos aprendidos y aplicados en los problemas que ilustran los conceptos, y otras más sofisticadas y profesionales, cuyos métodos de cálculo en algunos casos sólo pueden ser vislumbrados por el alumnado en estas etapas iniciales de su formación, que se irá completando en cursos sucesivos, pero que le permiten comprender el inmenso potencial de cálculo disponible en la actualidad, y su aplicación a problemas reales.

Por tanto, para ambos bloques temáticos (flujo en presión y flujo en lámina libre), se ha hecho un diseño paralelo de aplicaciones, con un conjunto de herramientas sencillas, basadas en Excel, y el uso de modelos profesionales: EPANET para flujo en presión e IBER para flujo en lámina libre, cuyo encaje en la asignatura y su vinculación con las prácticas se expone en los siguientes apartados.

En el caso de las aplicaciones Excel se ha optado por un diseño absolutamente plano y explícito, sin macros, botones o una "shell" que enmascare los cálculos que se están realizando, porque lo que se busca es que los cálculos sean fácilmente reconocibles y trazables. Esto es una decisión consciente. El grupo cuenta con un importante sustrato informático y se podría haber desarrollado mediante programación en VBA un conjunto de aplicaciones "bonitas", pero no es eso lo que se busca. 
En el caso de los modelos profesionales, se opta por casos sencillos para realizar una primera aproximación a su uso profesional. El trabajar con ejemplos relativamente simples permite además que los alumnos evalúen de forma crítica sus resultados y evita así que se conviertan en una "caja negra".

\subsection{APLICACIONES VINCULADAS AL FLUJO EN PRESIÓN}

El acercamiento habitual al flujo en presión comienza con el concepto de energía, pendiente motriz, disipación continua de energía y pérdidas locales. Las conocidas ecuaciones que cuantifican estos conceptos son, por ejemplo, la ecuación de Darcy-Weisbach, las expresiones de Colebrook-White o la forma simplificada de Swamee-Jain para la evaluación de la disipación continua y las expresiones para la evaluación de disipación local de energía.

Se ha desarrollado una aplicación Excel para la evaluación de las pérdidas continuas en un segmento de tubería utilizando para el coeficiente de Darcy-Weisbach la expresión de SwaneeJain (Figura 1). Las variables implicadas son la disipación de energía a lo largo del segmento, el caudal, las características de la tubería, como su diámetro, su rugosidad y su longitud, y las características del fluido, como su viscosidad o su densidad. La aplicación permite calcular una variable conocido el resto. Tienen interés académico y práctico:

- Calcular la disipación de energía. Es un cálculo directo, primera aplicación de las ecuaciones.

- Calcular el caudal circulante. Es un cálculo implícito, que requiere el uso de las aplicaciones de optimización de Excel.

- Calcular el diámetro adecuado. Es un cálculo implícito, y además la solución debe extraerse de un conjunto finito de diámetros comerciales.

El siguiente paso es la incorporación de pérdidas localizadas (por codos, válvulas o elementos similares). Esto no supone una dificultad añadida, pero permite percibir como, a igualdad de dimensiones en la tubería, la incorporación de estos elementos hace descender el caudal 
circulante. La aplicación permite además calcular los términos energéticos (cota, presión, velocidad) en los diversos puntos de una conducción.
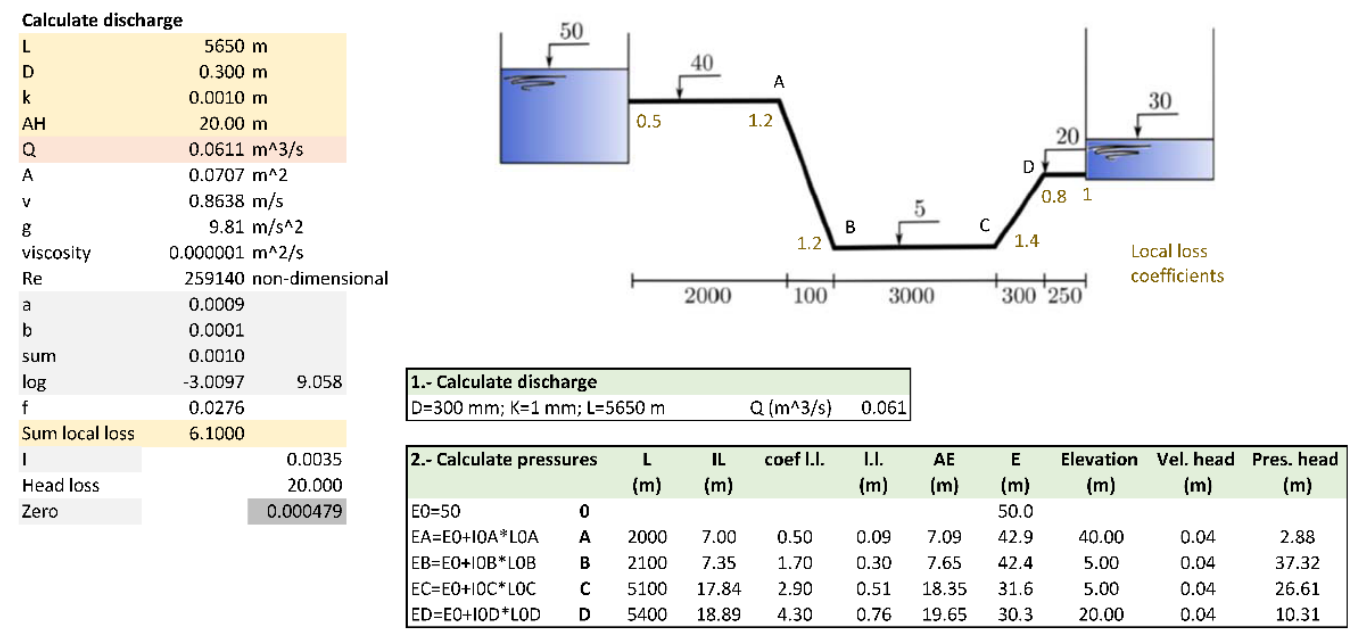

Figura 1. Aplicación Excel para el cálculo de pérdidas en un segmento de tubería.

La incorporación de impulsiones es el siguiente paso en el cálculo de conducciones a presión. Se introduce el concepto de curva resistente de la instalación y de curva característica de la impulsión, para definir un punto de funcionamiento (Figura 2). Se analiza la presión aguas arriba y aguas abajo de la bomba para distintas ubicaciones para ilustrar el concepto de NPSH y detectar si puede haber problemas de cavitación.

El siguiente paso, y el último en esta batería de aplicaciones simples, es el análisis de redes, que se ilustra con el problema de los tres depósitos (Figura 3). Utilizando los procedimientos de optimización de Excel se impone la ecuación de conservación de la energía en el nudo manteniendo una distribución consistente de caudales. En esta aplicación, que es el preámbulo del siguiente bloque, en el que se usará un modelo profesional, se introduce también el efecto del tiempo. Los caudales circulantes van llenando y vaciando los depósitos y esto modifica la distribución de flujos. 

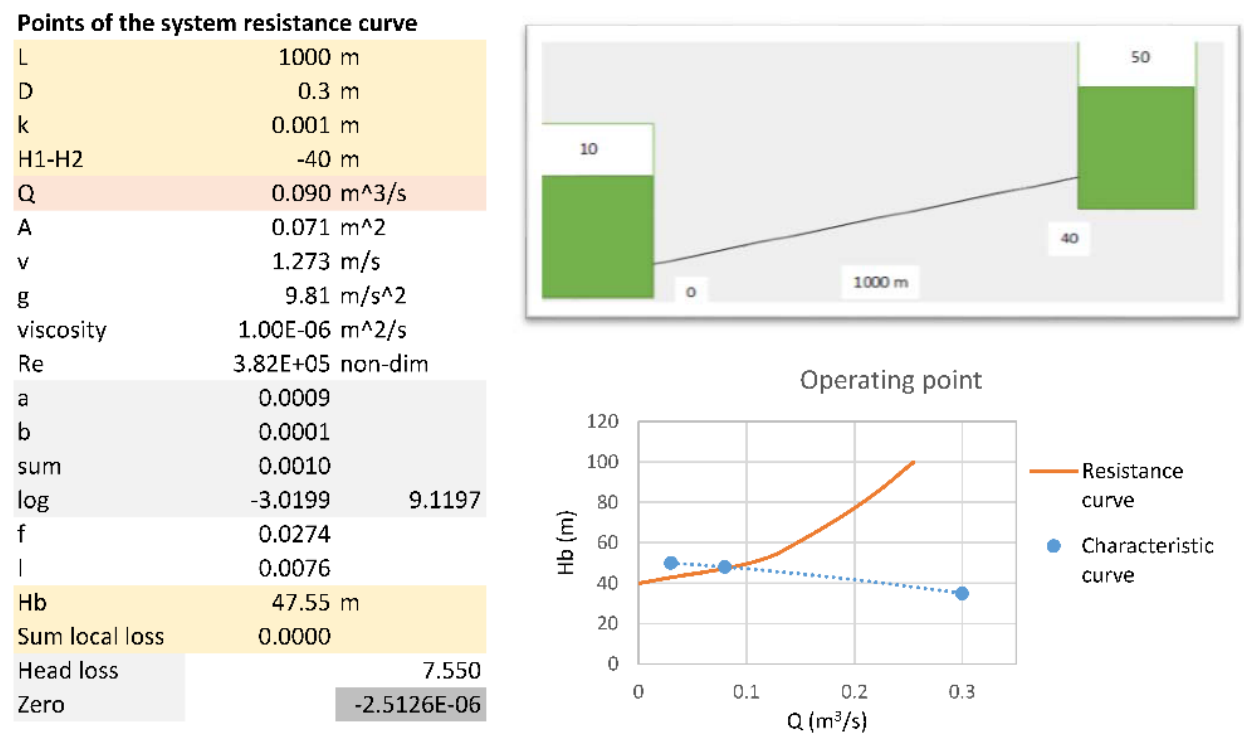

Figura 2. Aplicación Excel para el cálculo de una impulsión.
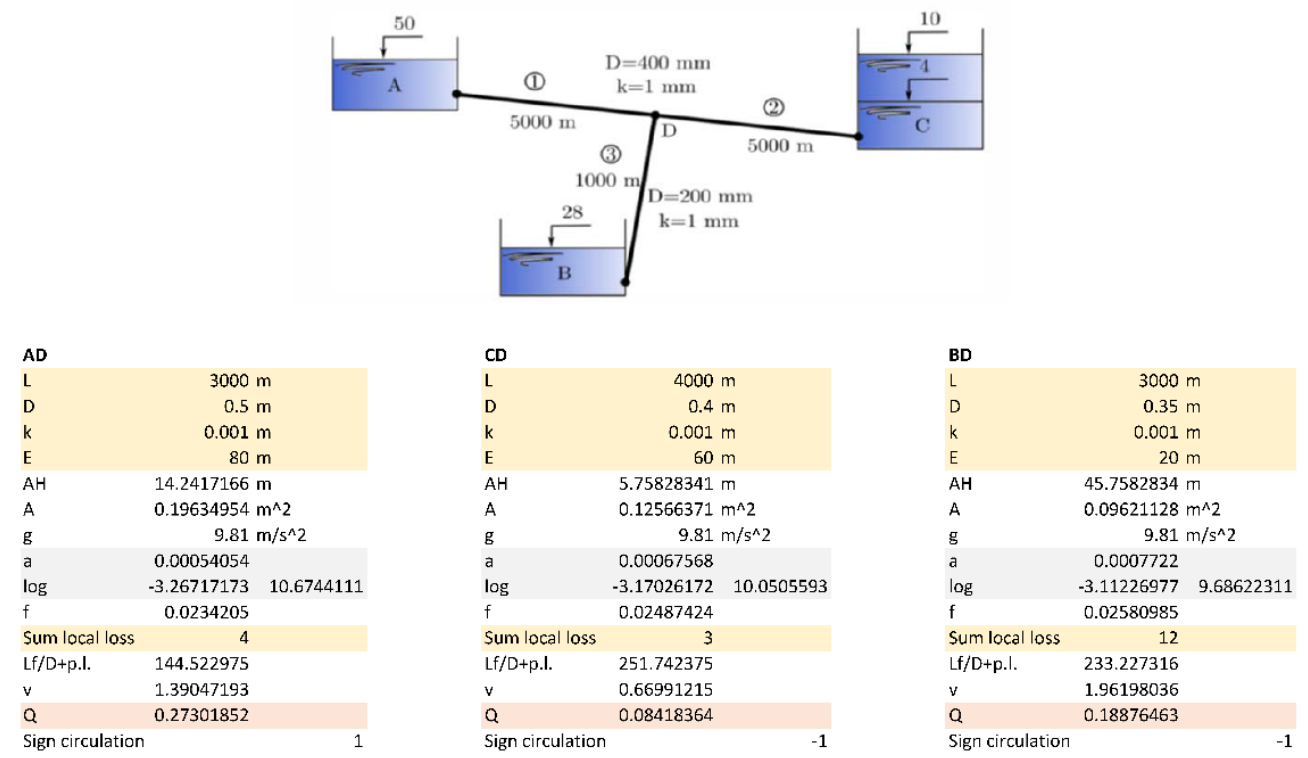

Figura 3. Aplicación Excel para el cálculo del problema de los tres depósitos. 
Una vez los alumnos han asimilado y manejado los conceptos simples es importante que manejen un programa profesional de cálculo de conducciones a presión. El estándar actual es EPANET (https://www.epa.gov/water-research/epanet), sobradamente conocido. Los alumnos reciben formación interactiva sobre este programa analizando problemas básicos, como algunos de los citados en los párrafos anteriores, y posteriormente se les emplaza a modelizar un sistema de conducciones de características reales. Para ello, se utiliza la infraestructura de investigación existente en el CITEEC (laboratorio adjunto a la Escuela de Ingeniería Civil), que incluye una conducción de fundición de características similares a la del abastecimiento de la ciudad de A Coruña (Figura 4). La conducción está ampliamente instrumentada con sensores de presión y caudalímetros, y el objeto de la práctica es introducir en EPANET la conducción, establecer unas condiciones de flujo y comprobar que el régimen de caudales y la distribución de presiones modelizado coincide con la realidad.

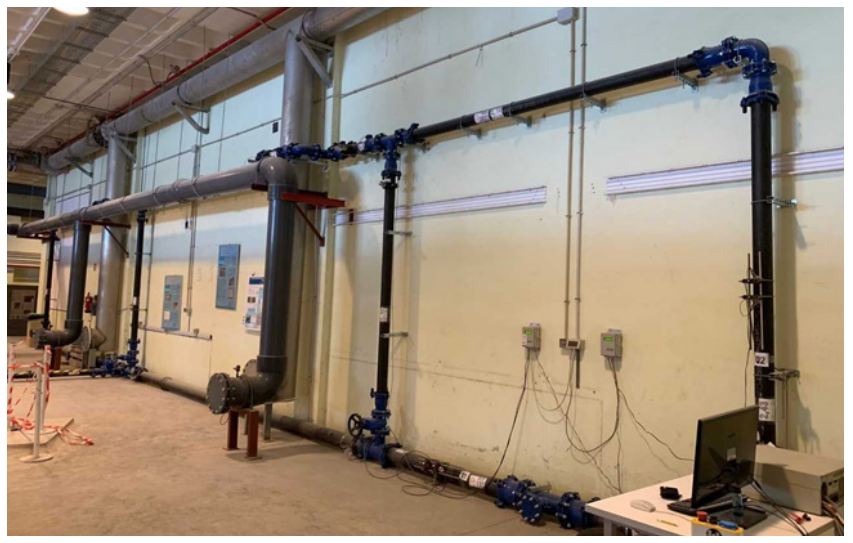

Figura 4. Sistema de conducciones del CITEEC utilizado en la práctica.

\subsection{APLICACIONES VINCULADAS AL FLUJO EN LÁMINA LIBRE}

En esta asignatura introductoria se explica con cierto detalle el movimiento permanente y uniforme y también el movimiento permanente gradualmente variado. Para ello, se acompañan Ios materiales teóricos con pequeñas aplicaciones Excel, que permiten reproducir resultados observados en el canal del laboratorio de la Escuela de Ingeniería Civil (Figura 5). Dicho canal 
tiene una longitud de $15 \mathrm{~m}$, una anchura de $50 \mathrm{~cm}$ y un calado máximo de $50 \mathrm{~cm}$, y tanto el caudal como la pendiente se pueden variar. Adicionalmente, dispone de un depósito de nivel variable en su extremo de aguas abajo que permite imponer diversas condiciones de contorno, para simular distintos tipos de perfil en la superficie libre.

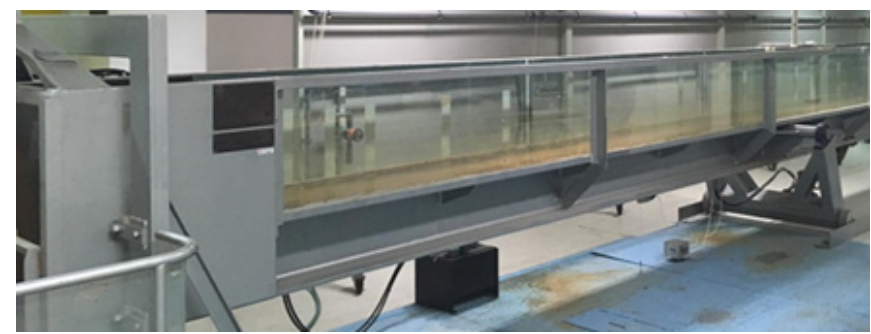

Figura 5. Canal del laboratorio docente de la Escuela de Ingeniería Civil.

La primera aplicación presenta las características básicas de un canal con diversas geometrías, como son su calado normal, su calado crítico y variables derivadas de éstas como su velocidad o el calado conjugado del calado normal. Se proponen secciones rectangulares, triangulares, trapeciales y circulares. Esta hoja permite interactuar con el canal del laboratorio, imponiendo en el mismo un calado constante mediante la manipulación de la condición de contorno, y comprobar que ese calado cumple efectivamente la ecuación del calado normal para diversos caudales y pendientes. Dado que la sección del canal es rectangular, se usa la pestaña correspondiente a esa geometría, que es la que se reproduce en la figura 6 .

La última aplicación sencilla es la que permite dibujar curvas de remanso, y se usa para reproducir las curvas que se observan en el canal del laboratorio (Figura 7). Esta aplicación permite por tanto "ensayar" (en forma de simulación), antes, durante y después de las prácticas en el laboratorio presencial. El alumnado impone diversas pendientes, caudales y condiciones iniciales, calcula los calados normal y crítico y dibuja sobre un papel las curvas observadas en el laboratorio, extrayendo de ellas un máximo de parámetros (el calado crítico 
si hay un vertido libre, o el calado normal si se llega a una condición de calados constantes, por ejemplo).

Paralelamente, la situación se reproduce en una pantalla en el propio laboratorio utilizando la aplicación, en la que se fijan los parámetros del flujo, se indica el sentido de integración (hacia aguas arriba en régimen lento y hacia aguas abajo en régimen rápido) y el intervalo de integración. La aplicación integra la ecuación de las curvas de remanso mediante un algoritmo de Runge-Kutta de cuarto orden, que se explicita en la hoja y que ha sido analizado previamente en las clases teóricas.

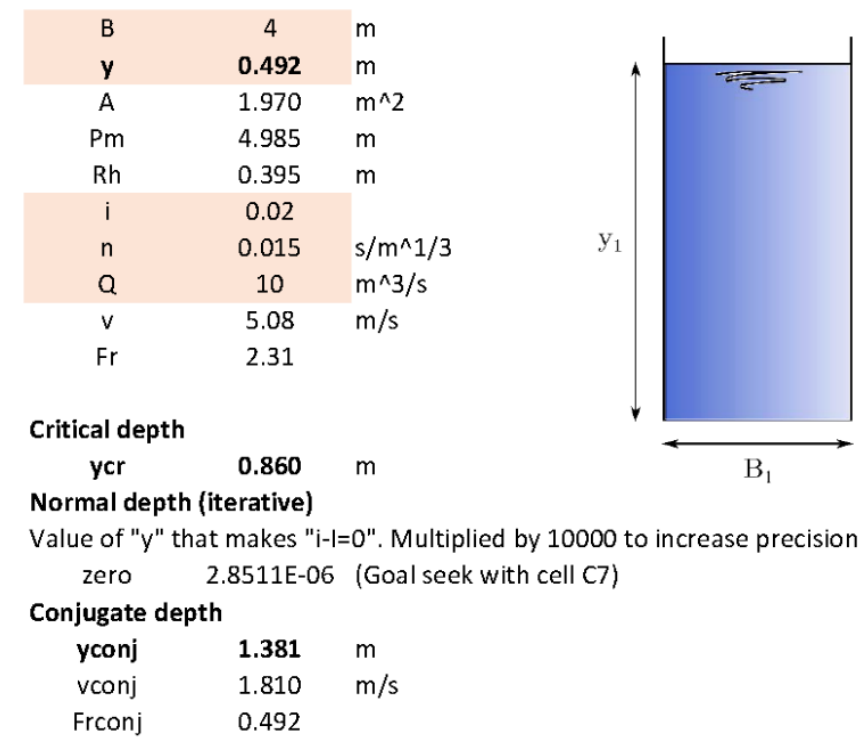

Figura 6. Aplicación Excel para el cálculo del calado normal, crítico y conjugado en una sección rectangular.

El resultado es una gráfica donde se presentan el fondo del canal, el calado normal, el calado crítico, el perfil de la superficie libre y cada uno de los calados conjugados (Figura 7). La aplicación permite incluir los datos medidos en el canal y comprobar el error medio cuadrático entre los datos medidos y los datos calculados, y calcular el coeficiente de Manning que optimiza el ajuste, utilizando la herramienta Solver. 

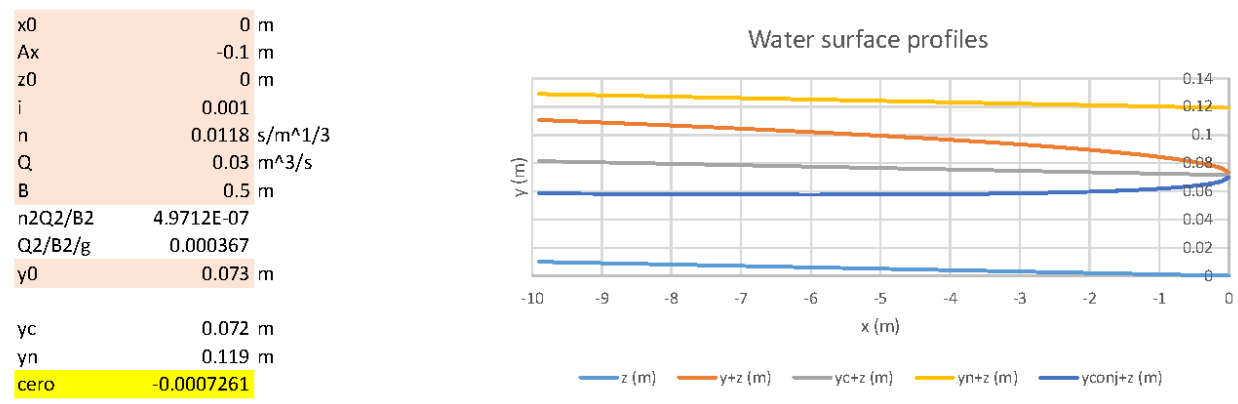

\begin{tabular}{|c|c|c|c|c|c|c|c|c|c|c|}
\hline$x(m)$ & $z(m)$ & $y(m)$ & $y+z(m)$ & $y c+z(m)$ & $y n+z(m)$ & $\mathrm{Fr}$ & $y \operatorname{conj}(\mathrm{m})$ & $y \operatorname{conj}+z(m)$ & $\begin{array}{c}\text { Measured } \\
y(\mathrm{~m})\end{array}$ & $\begin{array}{c}\text { MSE } \times 10^{\wedge} 6 \\
13091.3\end{array}$ \\
\hline 0 & 0 & 0.073 & 0.073 & 0.072 & 0.119 & 0.971 & 0.070 & 0.070 & 0.03 & 0.0018 \\
\hline-0.1 & 0.0001 & 0.076 & 0.076 & 0.072 & 0.119 & 0.912 & 0.067 & 0.067 & & 0 \\
\hline-0.2 & 0.0002 & 0.078 & 0.078 & 0.072 & 0.120 & 0.887 & 0.066 & 0.066 & & 0 \\
\hline-0.3 & 0.0003 & 0.079 & 0.079 & 0.072 & 0.120 & 0.868 & 0.065 & 0.065 & & 0 \\
\hline-0.4 & 0.0004 & 0.080 & 0.080 & 0.072 & 0.120 & 0.853 & 0.064 & 0.065 & & 0 \\
\hline-0.5 & 0.0005 & 0.080 & 0.081 & 0.072 & 0.120 & 0.840 & 0.063 & 0.064 & & 0 \\
\hline-0.6 & 0.0006 & 0.081 & 0.082 & 0.072 & 0.120 & 0.829 & 0.063 & 0.063 & & 0 \\
\hline-0.7 & 0.0007 & 0.082 & 0.082 & 0.072 & 0.120 & 0.819 & 0.062 & 0.063 & & 0 \\
\hline-0.8 & 0.0008 & 0.082 & 0.083 & 0.072 & 0.120 & 0.810 & 0.062 & 0.063 & & 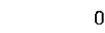 \\
\hline-0.9 & 0.0009 & 0.083 & 0.084 & 0.072 & 0.120 & 0.802 & 0.061 & 0.062 & & 0 \\
\hline-1 & 0.001 & 0.083 & 0.084 & 0.073 & 0.120 & 0.794 & 0.061 & 0.062 & & 0 \\
\hline
\end{tabular}

Figura 7. Aplicación Excel para el cálculo de curvas de remanso.

De un modo paralelo a lo que se planteó en el caso del flujo en presión, una vez asimilados los conceptos básicos en el laboratorio y mediante las aplicaciones sencillas, se da un paso hacia los modelos profesionales mediante la presentación y utilización del código de flujo bidimensional Iber (http://www.iberaula.es/). El modelo Iber permite el análisis de tramos de ríos o estuarios y tiene miles de usuarios registrados. Es un desarrollo de las universidades de A Coruña y Politécnica de Catalunya, junto con el CEDEX y el CIMNE (Bladé et al. 2014). A pesar de que es un modelo de flujo 2D, se han preparado una serie de aplicaciones sencillas para el primer contacto de los alumnos con el modelo, que permitan visibilizar el efecto de cambios de pendiente, cambios de sección, escalones y otras singularidades. Se trata de ejemplos que habitualmente no pueden ser realizados en el canal hidráulico de un laboratorio docente, por la complejidad de la geometría. La aplicación permite "ensayar" virtualmente estas geometrías de forma análoga a como se realizaría en el laboratorio convencional (presencial). Las distintas geometrías están documentadas en la publicación docente de Cea et al. (2018). A medida que el alumnado maneja la aplicación con estas sencillas geometrías 
se adentra en el uso del modelo. En las últimas clases del curso se explica, a título divulgativo, el potencial de cálculo del modelo sobre ejemplos de casos reales de aplicación en todo el mundo.

\section{CONCLUSIONES}

En la evaluación realizada al final del curso, la mayoría de los estudiantes señalan que han tenido una experiencia de aprendizaje agradable y que han estado motivados para realizar las actividades del curso. Estos resultados positivos han animado a los autores a compartir su experiencia. Los cursos introductorios a la mecánica fluidos son comunes a diversas titulaciones universitarias dentro de la ingeniería (mecánica, civil, etc.). Es sin embargo innegable que la introducción de nuevas metodologías activas en el aula universitaria requiere un esfuerzo por parte del profesorado, que debe producir nuevo contenido de calidad y adaptado a la nueva estrategia de enseñanza, con un apoyo institucional limitado. Para facilitar su aplicación 0 adaptación a otros cursos y/o titulaciones, las herramientas desarrolladas se han puesto a disposición de la comunidad en Zenodo (https://doi.org/10.5281/zenodo.3522670). Los dos softwares profesionales empleados en los ejemplos son de distribución gratuita y pueden obtenerse en sus respectivas webs. Se busca con ello eliminar las barreras externas habitualmente identificadas en la integración de tecnología en el aula, como las limitaciones de tiempo o la falta de recursos (Ertmer 1999). El implantar la metodología no conlleva ningún coste económico y las herramientas suministradas pueden utilizarse tal y como se presentan.

Cabe señalar asimismo que todas las aplicaciones Excel son fácilmente adaptables, pudiéndose variar fácilmente las geometrías de acuerdo con las instalaciones de laboratorio disponibles, o generar nuevos casos a partir de ellas. Siguiendo la metodología docente propuesta, es posible asimismo incorporar otras herramientas para profundizar en temas que puedan ser de mayor interés, dependiendo del enfoque de la asignatura (por ejemplo, la herramienta profesional "Allievi” para el cálculo de transitorios en tuberías). 


\section{REFERENCIAS}

Berrett, D. (2012). How "Flipping" the Classroom Can Improve the Traditional Lecture. Educ. Dig. Essent. Read. Condens Quick Rev, 78 (1), pp. 36-41.

Bladé et al. (2014). Iber: Herramienta de simulación numérica del flujo en ríos. Revista Internacional de Métodos Numéricos para Cálculo y Diseño en Ingeniería, 30 (1), pp. 1 10.

Cea, L., Bermúdez, M. \& Sobral, B. (2018). Cálculo de Curvas de Remanso y Fenómenos Locales Con Iber, Servizo de Publicacións da Universidade da Coruña, A Coruña, https://ruc.udc.es/dspace/handle/2183/20804?locale-attribute=es

Ertmer, P.A. (1999). Addressing first- and second-order barriers to change: Strategies for technology integration, Educ. Tech. Res. Dev., 47 (4), pp. 47-61.

Gibbs, G. \& Simpson, C. (2005). Conditions under which assessment supports students' learning. Learn. Teach. High Educ. 1, pp. 3-31. http://eprints.glos.ac.uk/3609/

Marqués Andrés, M. (2016). Qué hay detrás de la clase al revés (flipped classroom), ReVisión 9 (2016), no. 3, 2.

Puertas et. al. (2016). Apuntes De Ingeniería Hidráulica, Fundación de la Ingeniería Civil de Galicia, A Coruña.

UNESCO (1983). Experimental facilities in water resources education. A contribution to the International Hydrological Programme. UNESCO Technical Papers in Hydrology No. 24.

Wachs et al. (2018). Successfully flipping a fluid mechanics course using video tutorials and active learning strategies: Implementation and assessment. En: Proceedings of the 2018 American Society for Engineering Education Annual Conference \& Exposition. Salt Lake City, UT. 
